\title{
The Study of Location Specific Lane Change Impacts on Traffic Delay using Extended Cell Transmission Model
}

\author{
Susilawati Susilawati ${ }^{1, *}$, Kar Yow Tan ${ }^{1}$, Kamal Md Abdus Samad ${ }^{1}$, and Christina Ng$^{1}$ \\ ${ }^{1}$ Department of Civil Engineering, Monash University, Bandar Sunway, 47500 Subang Jaya, \\ Malaysia
}

\begin{abstract}
This paper comprehensively evaluates the influence of location specific lane change executions on delay and queue length. The extended macroscopic Cell Transmission Model (CTM) based lane change model has been developed by adopting the formulations of diverging and merging in one-way traffic flow. The extended CTM model uses a pre-determined lane change rate and traffic parameters including free flow speed, jam density, cell length and time-step while the inflow parameters were generated form real traffic data collected in Bandar Sunway, Malaysia. The results demonstrate that the lane change maneuver approaching the exit of intersection incurs the highest delay. From real observation, drivers have high tendency to perform mandatory lane changing compared with discretionary lane change in urban arterials. The results can be further used to develop lane change assistance model to improve the traffic flows.
\end{abstract}

\section{Introduction}

The World Bank Malaysia Economic Monitor: Transforming Urban Transport report 2015 has revealed a statistic that the economic losses caused by traffic congestion in Kuala Lumpur, urban metropolitan city of Malaysia is approximately RM20 billion per annum or RM 54 million per day [10]. This numerical cost figure is collected from the cost of lost productivity while drivers stuck in traffic jam, delay cost of traffic network, cost spent on the fuel consumption, environmental cost due to tailpipe exhaust fume emission, etc. Another statistic conducted by World Bank shows that Kuala Lumpur residents spend 250 million hours per year stuck in traffic gridlock with $29 \mathrm{~km} / \mathrm{h}$ slower travelling speed during morning peak hours [10]. The statistic results show that how severe traffic congestion decrease the traffic flow efficiency and constitute economic and environmental degradation. Other hidden costs include adverse health effects from physical inability and high stress levels of drivers stuck in traffic congestion.

Lane changing maneuver is one of the main factors that aggravate traffic congestion due to increasing queue length and delay in travel time [16]. Lane change also imposes road

*Corresponding author: susilawati@monash.edu 
safety hazards where traffic accidents are frequently occurred at weaving sections and interchanges [18].

A macroscopic traffic simulation model is proposed to study the lane changing behavior in multi-lane roads as it requires less amount of data and is more computationally efficient compared with microscopic model. The required data for macroscopic models can be easily obtained through standard monitoring infrastructures such as video camera and traffic detectors. Macroscopic simulation model can provide a global perspective of various traffic scenarios. This research project aims to study the impacts of lane change execution on traffic delay and queue length on a two-lane one-way urban arterial link. The effects of location of lane change are investigated.

Extended model of Cell Transmission Model (CTM) is developed to evaluate the traffic performance. The objectives of this research are follows:

(1) To develop an extended CTM by adopting di verging and merging formula.

(2) To evaluate the effects of lane change execution at different road segments on delay and queue length.

Given some constraints, this study focuses on effects of lane change execution at different road sections but neglect driver's lane change decision making process. The results indicate that lane changing execution approaching the exit of intersection incurs highest delay and queue length and suggest the close relationship between lane change occurrences with density in origin lane and target lane. The results can be further used to develop a lane change assistance system that may recommend location specific lane change behavior on a link to improve the traffic flows with minimum delay.

\section{Literature Review}

\subsection{Previous studies on macroscopic lane change models}

Microscopic traffic flow modelling is concerned with motion characteristics such as acceleration, deceleration and lane change of single vehicle in traffic stream [4]. Microscopic model analyses the movement of individual vehicles and interaction between a vehicle with another vehicle on different features of road [11]. Microscopic simulation model is capable of analysing traffic behaviour under different lane configurations, traffic compositions and traffic flow conditions as well as capture different vehicle classes. The examples of microscopic models include car-following models, lane change model, route choice model, etc. Most microscopic models are stochastic due to the difficulties to predict driver behaviour with absolute certainty in real life situation. However, the large number of vehicle specific parameters and complex computational inputs impede the use of microscopic simulation model

At macroscopic level, Lighthill and Whitham [20] assumed that traffic streams flow is comparable to fluid streams. Fig. 1 below shows the macroscopic fundamental diagram that describes the relationship between flow $(\mathrm{q})$, density $(\mathrm{k})$ and average speed $(\mathrm{u})$ of a traffic stream. The flow increases as the number of vehicles in the stream increases until the traffic volume reaches the maximum capacity where traffic congestion starts to develop. Afterward, the flow decreases towards zero so does the average speed until the traffic network reaches a gridlock state. 


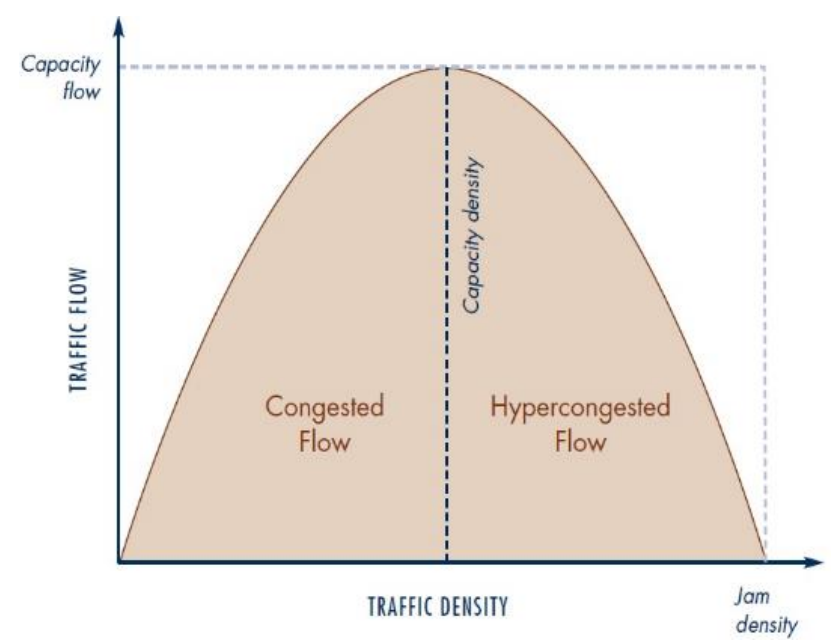

Fig. 1. Macroscopic Fundamental Diagram [3].

Lane change is defined as "a driving maneuver that transfer a vehicle from one lane to adjacent lane in the same direction of travel. In the well-known LWR model developed by Lighthill and Whitham [20] considers lane changing vehicles as an instantaneous accelerated fluid in single lane which does not consider car following behavior.

Lane change maneuver can be classified into two types, namely, mandatory and discretionary. Mandatory lane change (MLC) occurs when the drivers are required to execute lane change to get on appropriate path to their destination [5]. Drivers have tendency to perform MLC when there are obstructions or lane blockages ahead of the subject vehicles and taking an exit off ramp [24]. Discretionary lane change (DLC) occurs when drivers change to the adjacent lane that offers better traffic conditions to attain desired speed [1].

Several studies have analyzed the impact of lane change on overall traffic performance. Under congested traffic conditions, lane changing maneuvers reduced the discharge rate and triggered shockwaves and as a result affect the traffic operational efficiency (i.e. capacity reduction, delay) $[2,6,9]$. In addition to that, lane change vehicles are a major cause of collision and has brought significant impacts on traffic safety $[8,22]$. Accidents either minor or major, tends to happen in these areas (i.e. interchanges, weaving sections) which causes subsequent loss of lives. When the lane changing maneuvers occurs, speeds across lanes can be synchronized which results in a gain of speed advantage and travel time [14].

Laval and Danganzo [19] developed a hybrid approach that combines the strong points of macroscopic and microscopic model. They model each lane as a separate kinetic wave stream and devise a LWR model by extending single lane to multiples lanes. Cheu et al. [5] proposed a CTM macroscopic model for simulation of lane change between queues to estimate the average queue length. The drawback of the model is only discretionary lane change is considered. Jin [13] introduced a new lane change intensity variable from Edie's definition of traffic density. However, Jin [13] model focus on freeway segments with on and off ramps. Carey et al. [5] introduced a multilane CTM model by extending the single lane CTM model to investigate lane changing behaviour. Carey et al. [5] extend CTM to two adjacent lanes and model the vehicles movement between lanes using conservation equations. The experiment proposed by Carey et al. [5] states that the total inflow for two lanes are the same and considers single type of vehicle which are unrealistic in real life situation. 
From the review, it is clear that some assumptions were made to simplify the lane change model including the use of same total inflow for different lanes, and they only consider one type of vehicle. To fill the research gaps, the refined CTM based lane change model is necessary, which is addressed in this paper. Specifically, an extended CTM based lane change model is developed using real inflow traffic data collected from video camera and considering two types of vehicles, passenger car and truck. To simulate lane change execution in a two-lane arterial road, the diverging and merging formula with the predefined rate of lane change is incorporated to the proposed model.

\section{Methodology}

\subsection{Cell transmission model (CTM)}

Cell Transmission Model (CTM) provides discrete approximation to hydrodynamic or kinematic wave model proposed in LWR model [20]. CTM is a macroscopic model that simulate traffic flows at every time step by dividing the road sections into homogenous cells, numbered from upstream cell " $i$ " to downstream cell " $i+1$ " as illustrated in Error! Reference source not found. It is assumed that all the vehicles in downstream cell is advanced to downstream cells under light traffic condition.

$$
\stackrel{f_{i-1}(t)}{\longrightarrow} \underset{\text { cell } i-1}{n_{i-1}(t)} \stackrel{f_{i}(t)}{\longrightarrow} \underset{n_{i}(t)}{\text { cell } i} \stackrel{f_{i+1}(t)}{\longrightarrow} \underset{\text { cell } i+1}{n_{i+1}(t)} \stackrel{f_{i+2}(t)}{\longrightarrow}
$$

Fig. 2. Flow Advancement Diagram of CTM [25].

The length of each cell, $L$ is the product of free flow speed, $v$ and the duration of each time step, $t$ as shown in Equation 1.

$$
L=v t
$$

The holding capacity $\left(N_{i}(t)\right)$ of cell " $i$ " at time $t$ is the product of jam density, $k_{j a m}$ and cell length as listed in Equation 2.

$$
N_{i}(t)=k_{j a m} L
$$

The flow capacity, $Q$ is the product of saturation flow rate, $s$ and the duration of each time step as formulated in Equation 3.

$$
Q=s t
$$

The density of cell at each time step, $n_{i}$ is updated by adding the number of incoming vehicles and deducting the number of outgoing vehicles as shown in Equation 4.

$$
n_{i}(t+1)=n_{i}(t)+f_{i}(t)-f_{i+1}(t)
$$

CTM can accommodate different traffic conditions including light traffic, bottleneck and oversaturated traffic condition. In light traffic condition, the flow is the number of vehicles in cell " $i-1$ " at time $t$ whereas the flow is equal to saturation flow rate in cell " $i$ " at time $t$ in bottleneck traffic condition. In oversaturation traffic condition, the flow is equal to the amount of available space in cell " $i$ " at time $t$. The ratio of $W$, backward shockwave speed 
to $\mathrm{v}$, free flow speed is less than 1 in oversaturated condition [23]. The flow formulas of three traffic conditions are listed in Equation 5.

$$
f_{i}(t)=\min \left\{n_{i}(t), Q_{i}(t), W / v\left[N_{i}(t)-n_{i}(t)\right]\right\}
$$

In oversaturated traffic condition, delay occurs when the vehicles are not able to travel to the next cell and remain in the existing cell. Delay, $d_{i}(t)$ is denoted as the additional time for the vehicle travel from current cell to the next cell and can be calculated using Equation 6.

$$
d_{i}(t)=n_{i}(t)-f_{i+1}(t)
$$

Total delays of whole road network can be computed by aggregating all the delays of each cell for all time steps as shown in Equation 7.

$$
\text { Total Delay }=\sum_{t} \sum_{i} d_{i}(t)
$$

The average delay per vehicle can be computed by dividing the summation of delay of all cells at each time step by the corresponding inflow as listed in Equation 8.

$$
\text { Average delay per vehicle }=\frac{\sum_{i} d_{i}(t)}{f_{i}(t)}
$$

Queue length is the summation of the density of all cells at each time step as shown in Equation 9.

$$
\text { Queue Length }=\sum_{i} n_{i}(t)
$$

\subsection{Extended CTM based lane change model}

To cater for the occurrence of spillback, Wang [23] proposed an improved model to the CTM: Conditional Cell Transmission Model (CCTM) in which diverging and merging activities were taking into account. The behavior of diverging and merging vehicles at multiple lanes road were adopted to model lane change phenomenon. As illustrated in Error! Reference source not found., Cell a diverges into Cell $\mathrm{b}$ and Cell $\mathrm{c}$, in accordance with the percentage of lane changing, $\alpha$ and $\beta$ respectively. For a two-lane road segment, if Cell a is located at the left lane, Cell b denotes through vehicles and Cell $\mathrm{c}$ denotes right turns vehicles. Conversely, if Cell a is located at the right lane, Cell b indicates left turns vehicles and Cell $\mathrm{c}$ indicates through vehicles.

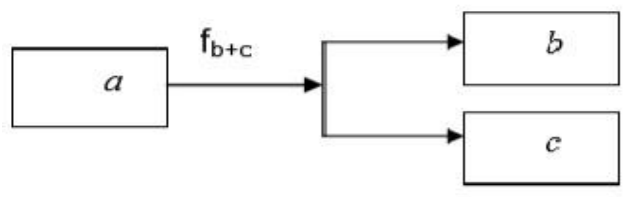

Fig. 3. Diverge in One-Way Flow [23]. 

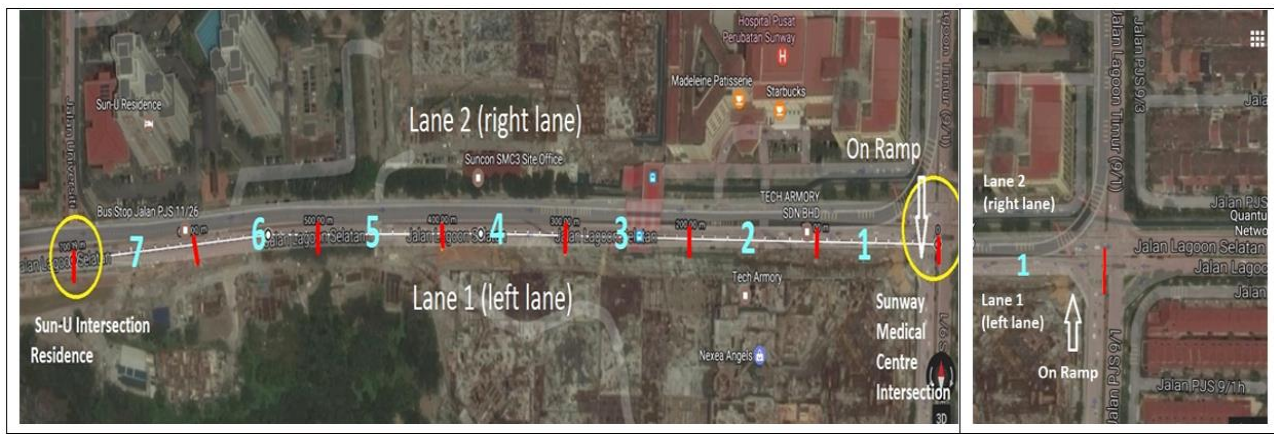

Fig. 4. Study Site and Intersection Geometry.

The diverge formula to calculate the total number of vehicles entering Cell b and Cell $\mathrm{c}$ is as follows.

$$
f_{b+c}(t)=\min \left\{\begin{array}{c}
n_{a}(t) \\
Q_{a}(t) \\
\left.\operatorname{Min}\left\{Q_{b}(t),\left[N_{b}(t)-n_{b}(t)\right]\right\} / \propto\right\} \\
\operatorname{Min}\left\{Q_{C}(t),\left[N_{c}(t)-n_{c}(t)\right]\right\} / \beta
\end{array}\right\}
$$

The equations of vehicles entering Cell b and Cell $\mathrm{c}$ are listed in Equations 10 and 11.

$$
\begin{aligned}
& f_{b}(t)=\propto \times f_{b+c}(t) \\
& f_{c}(t)=\beta \times f_{b+c}(t)
\end{aligned}
$$

As illustrated in Error! Reference source not found., Cell a and Cell b flow into Cell c. For a two-lane road segment where Cell a denotes left lane and Cell $b$ denotes right lane, the vehicles of both cells merge to a receiving lane, Cell $\mathrm{C}$ that can either entering left lane and right lane depends on the percentage of lane changing.

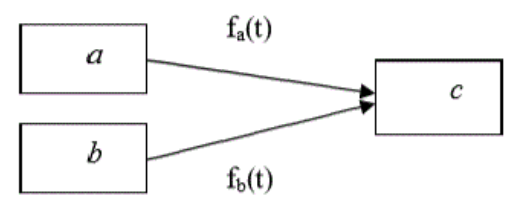

Fig. 5. Merge in One-Way Flow [23].

The merge formulas of vehicles in Cell a and Cell $\mathrm{b}$ flowing into Cell $\mathrm{c}$ are formulated as follows.

$$
\begin{aligned}
& f_{a}(t)=\min \left\{n_{a}(t), Q_{a}(t),\left[N_{c}(t)-n_{c}(t)\right]\right\} \\
& f_{b}(t)=\min \left\{n_{b}(t), Q_{b}(t),\left[N_{c}(t)-n_{c}(t)\right]\right\}
\end{aligned}
$$

The total vehicles flowing out from Cell a and Cell b merge into Cell $\mathrm{c}$ is formulated in Equation 13.

$$
f_{a+b}=f_{a}(t)+f_{b}(t)
$$




\subsection{Assumptions}

Few assumptions are made before developing lane change model. The drivers can execute lane change to the next adjacent cell in the target lane considering the minimum gap acceptance criterion. The lane change maneuver of all vehicles is feasible with no collision of subject vehicles with the preceding vehicles in the target lane. All vehicle travel at the same speed and have same priority in moving to downstream cells of origin lane or target lane regardless of vehicle types. The number of vehicles approaching first cell are exit in proportion to the number of vehicles in last cell or the maximum capacity of the last cell. The inflow of trucks is calculated by converting the demand of small-size truck into demand of passenger car by adopting the passenger car unit of 1.5. The passenger car unit of small- size truck with value of 1.5 denotes the size of the truck is approximately equivalent to 1.5 cars so that the inflow does not exceed the flow capacity of 4 vehicles per lane for each time-step.

\subsection{Model development}

Based on proposed methodology and assumptions discussed in Section 3.2 and 3.3, the extended CTM model for two lane arterial road were developed. This model adopts diverging and merging formula with pre-determined lane change rate which indicate the percentage of number of vehicles that execute lane change from the origin lane to the target lane and the number of vehicles flow through origin lane. The queue formation, propagation and dissipation phenomena can be investigated by analyzing the density of each cell.

The traffic variables and road network parameters are listed as follows:

(1) Road length: $700 \mathrm{~m}$

(2) Free-flow speed, v: $51 \mathrm{~km} / \mathrm{h}$

(3) The time step is $7 \mathrm{~s}$.

(4) Cell length $(\mathrm{L})=100 \mathrm{~m}$, so there are 7 cells and 172 time steps consists of 171 time steps (7s) and the final time step in proposed extended CTM.

(5) Backward shock wave speed, w: $21.6 \mathrm{~km} / \mathrm{h}$ [12]

(6) Saturation flow rate, s: 1800veh/h/lane

(7) Jam density, $k_{\text {jam }}: 102 \mathrm{veh} / \mathrm{km} /$ lane

(8) The lane changing rate is $35 \%$ which is based on study by Knoop [17] which suggested the number of lane change per vehicle kilometer is typically 0.5 . This statistical data indicates that drivers execute lane changing on average one per two km travelled. Therefore, for the 700 meters road length, the lane change rate of $35 \%$ is adopted.

The values of built-in parameters such as free-flow speed, saturation flow rate and jam density for urban arterials are obtained from Highway Capacity Manual [21]. Based on Equation 2 and 3 above, the holding capacity, $\mathrm{N}$ and the flow capacity, Q are calculated as 10 vehicles and 4 vehicles respectively.

The total inflow of first cell is based on traffic data collection conducted at Jalan Lagoon Selatan, between Sunway Medicine Centre (Sun-Med) Intersection and Sun-U Residence Intersection situated at Bandar Sunway in Selangor, Malaysia. Error! Reference source not found. illustrates the aerial view of the study site and the intersection geometry. Based on traffic data, the total in flow of ramp, lane 1 and lane 2 are 203, 124 and 115 vehicles respectively. 
To investigate the influence location specific of lane change execution, lane change with varying density on queue length and delay, several lane change scenarios were developed.

\section{Scenario 1: Change lane as soon as possible}

The first case aims to investigate the impacts of lane change execution when drivers desire to change lane as soon as they enter the link. A lane change percentage of $35 \%$ is allocated to model the lane changing behavior between Cell 1 to Cell 2 from L1 to L2 and from L2 to L1 for all time steps.

\section{Scenario 2: Lane change execution increase linearly along the link}

The second case is to evaluate the traffic system performance when the proportion of lane change execution increase linearly as driver approach the exit. The rate of lane change increases linearly from $0 \%$ to $11.65 \%$ with $2.33 \%$ increment that gives a total lane change percentage of $35 \%$ travelling from the upstream cell to downstream cell for all time steps.

\section{Scenario 3: Change lane at the last cell, Cell 7}

The third case aims to study the effects of the abrupt lane change at the last cell. This occur when driver notice that the distance available to execute lane changing is becoming shorter towards an exit. A lane change percentage of $35 \%$ is allocated to model the lane changing behavior between Cell 6 to Cell 7 from L1 to L2 and from L2 to L1 for all time steps.

\section{Scenario 4: Effect of last cell density on lane change execution}

This experiment is designed to investigate the relationship between density and number of lane change particularly when it occurs at the last cell.
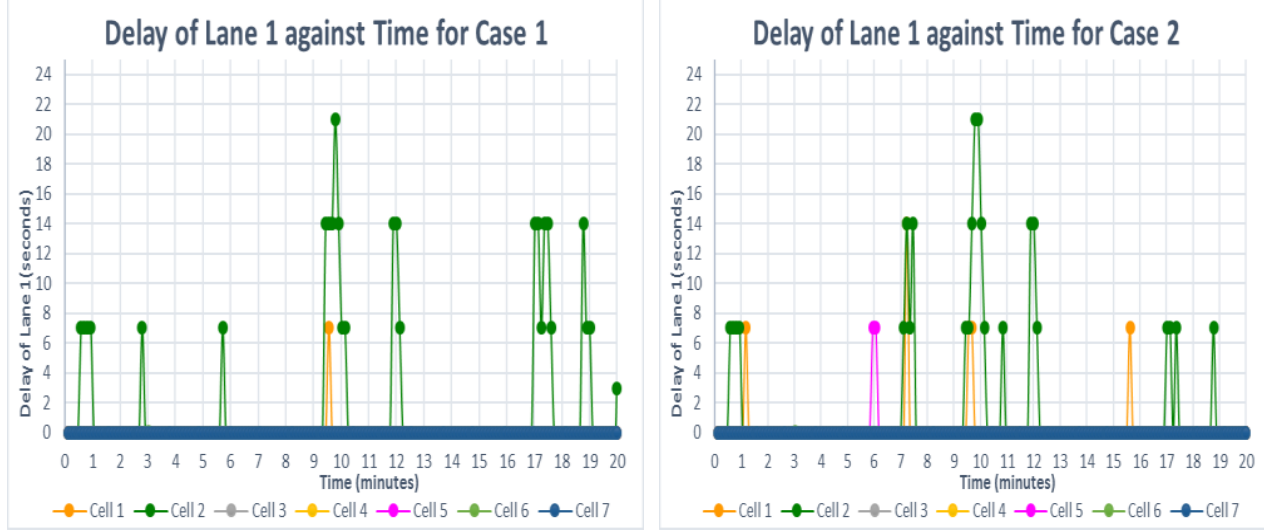


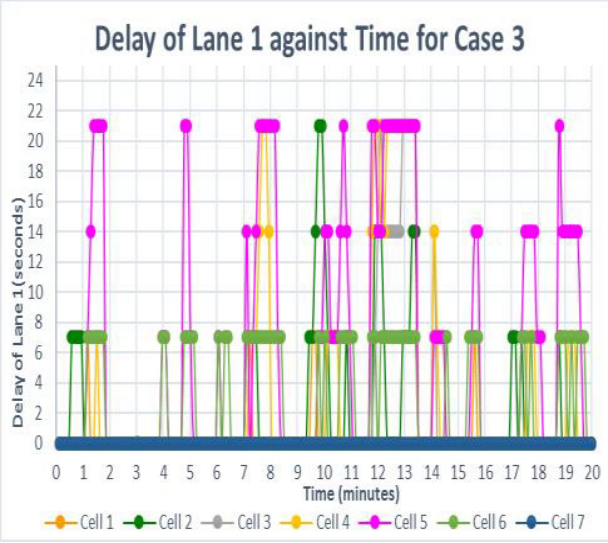

Fig. 6. Delay of Lane 1 against Time for Three Case.

Table 1. Result for Three Cases of Lane Changes at Different Road Sections.

\begin{tabular}{l|c|c|c|c|c|c|c|c|c}
\hline & \multicolumn{3}{|c|}{ Scenario 1 } & \multicolumn{3}{c|}{ Scenario 2 } & \multicolumn{3}{c}{ Case 3 } \\
\cline { 2 - 11 } & Ramp & $\begin{array}{c}\text { Lane } \\
1\end{array}$ & $\begin{array}{c}\text { Lane } \\
2\end{array}$ & Ramp & $\begin{array}{c}\text { Lane } \\
1\end{array}$ & $\begin{array}{c}\text { Lane } \\
2\end{array}$ & Ramp & $\begin{array}{c}\text { Lane } \\
1\end{array}$ & $\begin{array}{c}\text { Lane } \\
2\end{array}$ \\
\hline Total Delay (s) & 28 & 318 & 0 & 35 & 385 & 21 & 35 & 3399 & 21 \\
\hline $\begin{array}{l}\text { Maximum Average } \\
\text { Delay Per Vehicle } \\
\text { (s/veh) }\end{array}$ & 7 & 14 & 0 & 7 & 28 & 3.5 & 7 & 98 & 3.5 \\
\hline $\begin{array}{l}\text { Maximum Queue } \\
\text { Length (veh) }\end{array}$ & 4 & 23 & 14 & 4 & 22 & 18 & 4 & 29 & 17 \\
\hline
\end{tabular}

\section{Results and Discussions}

\subsection{The influence of specific location of lane change execution on delay and queue length}

As noted above in model parameters, the number of vehicles entering ramp, lane 1 and lane 2 are 203, 124 and 115 vehicles respectively. Based on Error! Reference source not found., Lane 1 has the highest values of total delay, maximum average delay per vehicle and maximum queue length. The result is reasonable, because Lane 1 accumulates the traffic flow of vehicles driving in the lane, the vehicles that executed lane change from Lane 2 and exit into Lane 1 and merged vehicles from ramp, thus greatly increase the density and delay. The ramp rank second highest total delay and maximum average per vehicles due to high traffic volume. Nevertheless, the queue length of ramp is short due to the its short road stretch of approximately $20 \mathrm{~m}$ that leave limited rooms for vehicles to stay in the cell. The low inflow of Lane 2 incurs the lowest total delay and maximum average delay per vehicle and slightly shorter queue length compared with Lane 1.

Three cases of lane change in varying road sections are compared. Case 3 has the highest total delay and maximum average delay per vehicle of 3399 seconds and 98seconds per vehicle respectively. The lane change maneuver at last cell results in density accumulation and queue formation at the early stage that greatly increase the density and queue length in downstream cells. The queue will eventually spillback along the link to Cell 1 . Due to the high density in cells along the link, the vehicles are not able to move to downstream cells and remained in the current cell thus leads to large delay and queue length. Case 2 has higher total delay and maximum average delay per vehicle compared with Case 1. The former 
defines the lane change is distributed along the link instead of the vehicle abruptly change lane at the first cell. That in turns reduce the queue length due to queue propagation.

Lane 1 is selected to evaluate the delay performance of entire network as it experiences the highest delay. From Error! Reference source not found., it was observed that delay of Lane 1 occurs on Cell 1 and 2 only for Case 1. The delay for Case 1 reached the peak around 9 minutes 40 seconds after the simulation period (timestep 84) with a value of 21 seconds. The lane change of vehicles at Cell 1 increases the flow entering Cell 2 . Hence, the vehicles of Cell 2 require additional time travel to the next cell. The vehicles are traveling smoothly from Cell 3 onwards that can be justified by the presence of no delay observed in other cells for the entire network. Another reason of Cell 2 of Lane 1 has high delay is because Lane 1 (Cell 2) that is located at the downstream of on-ramp receives the merging vehicles from onramp (Cell 1 Ramp).

In comparison to Case 1, Case 2 exhibits similar delay pattern with Lane 1 but with more delay distributed along Cell 1 . As the lane change is spread out along the link, the delay occurs further along the link resulting Cell 5 has a delay value of 7 seconds around 6 minutes. Based on Error! Reference source not found. above, all cells experience delay except Cell 7 for Case 3 concluded that the lane change at last cell increases the overall delay of the road network. The network reaches a steady constant delay of 21 seconds for Cell 5 from 12 minutes to 13.30 minutes indicates that the number of vehicles entering Cell 5 are exit in proportion to Cell 6.

\subsection{The influence of density on number of lane change}

The relationship between density and number of lane change particularly when it occurs at the last cell were investigated. The benchmark of the entry point and the exit point of Cell 7 were identified. The videotape is played back to track the movement between lanes of individual vehicle in Cell 7 record the he density of Cell 7at each time-step. In this case, the vehicles are considered as perform lane change maneuver when the drivers move from origin lane to the adjacent lane regardless of the types of lane change carry out (mandatory lane change or discretionary lane change). The numbers of lane change from Lane 2 to Lane 1 against density of Lane 1 and Lane 2 are investigated due to its higher values of the numbers of lane change. 


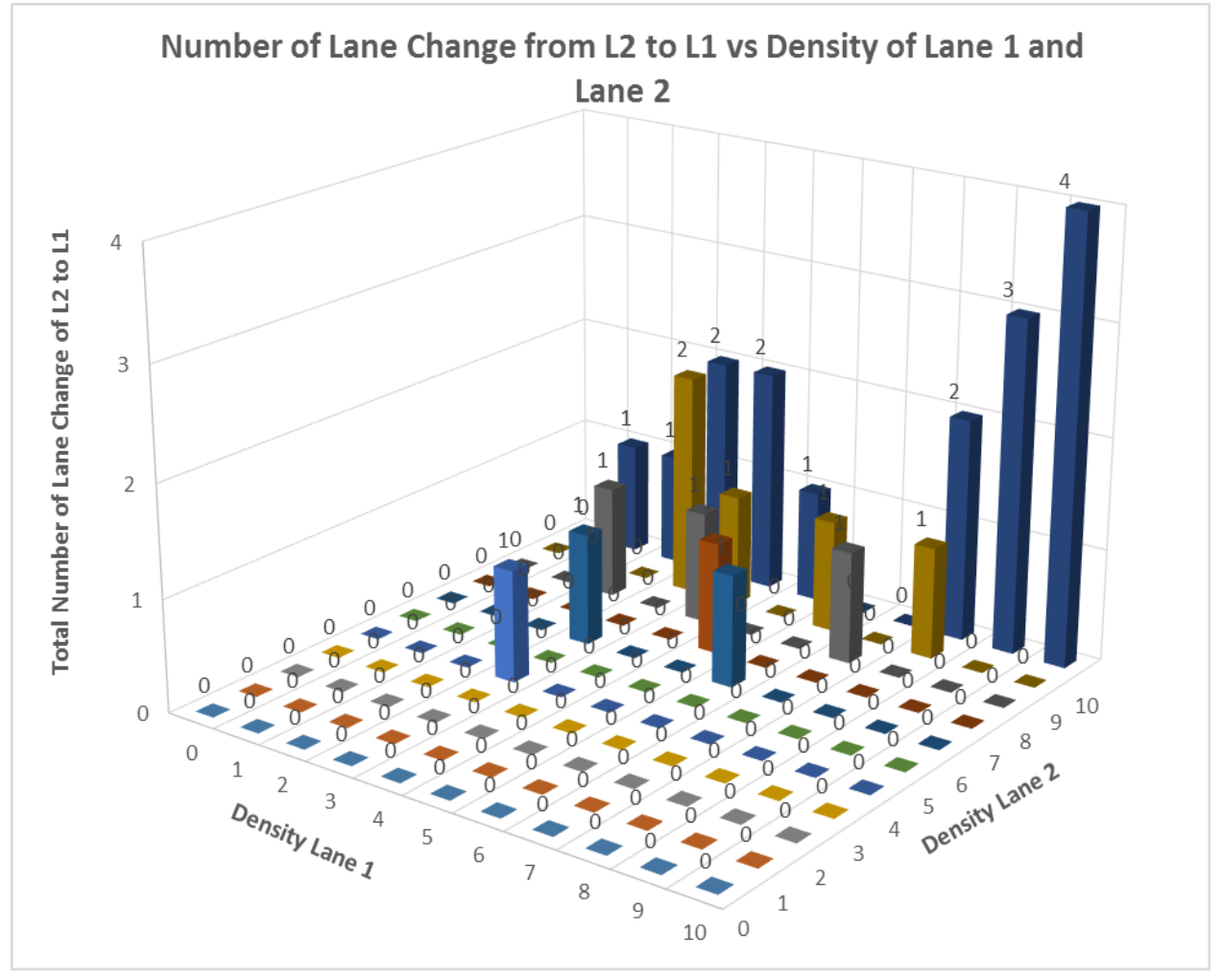

Fig. 7. Total Number of Lane Change from L2 to L1 against Density in Lane 1 and Lane 2.

Table 2. Total Number of Lane Change from L2 to L1 with Varying Density in Lane 1 and Lane 2.

\begin{tabular}{ccc}
\hline Density Lane 1 & Density Lane 2 & Number of Lane Change \\
\hline veh & veh & 1 \\
1 & 10 & 1 \\
2 & 8 & 1 \\
2 & 10 & 1 \\
3 & 4 & 1 \\
3 & 6 & 2 \\
3 & 9 & 2 \\
3 & 10 & 1 \\
4 & 8 & 1 \\
4 & 9 & 2 \\
4 & 10 & 1 \\
5 & 7 & 1 \\
5 & 10 & 1 \\
6 & 6 & 1 \\
6 & 9 & 1 \\
7 & 8 & 1 \\
8 & 9 &
\end{tabular}




\begin{tabular}{ccc}
8 & 10 & 2 \\
9 & 10 & 3 \\
10 & 10 & 4 \\
\hline
\end{tabular}

As displaced in Error! Reference source not found. above, the number of lane change from Lane 2 to Lane 1 reached the peak with a value of 4 when the density of both Lane 1 and Lane 2 have the maximum occupancy of 10 vehicles. The results show the aggressiveness of driver behavior to switch lane at urban arterials which can be further investigated in future research. Based on Error! Reference source not found. above, the number of lane changes increase with the density in origin lane (Lane 2) with a constant density in target lane (Lane 1) where the density in Lane 1 are 3, 4 and 8 vehicles. Besides, the number of lane change also increases with the density in target lane (Lane 1) for with a constant density in origin lane (Lane 2) where the density in Lane 2 are 8,9 and 10 vehicles.

Further analysis was conducted to determine the likelihood of lane change execution at urban arterials, the lane change behavior in Cell 7 were investigated. The videotape is played back to track the movement between lanes of individual vehicle in Cell 7 and after exiting cell 7. The lane changing maneuver of drivers are categorized as mandatory lane change if the drivers perform lane change in the last cell and travelling along the same lane after leaving Sun-U Residence Intersection. A driver is said to perform discretionary lane change if the driver carries out lane change in the last cell but performs lane changing again after exiting Sun-U Residence Intersection to reach his destination. The pictures of mandatory and discretionary lane changing execution from Cell 7 exiting Sun-U Residence Intersection are illustrated in Appendix.

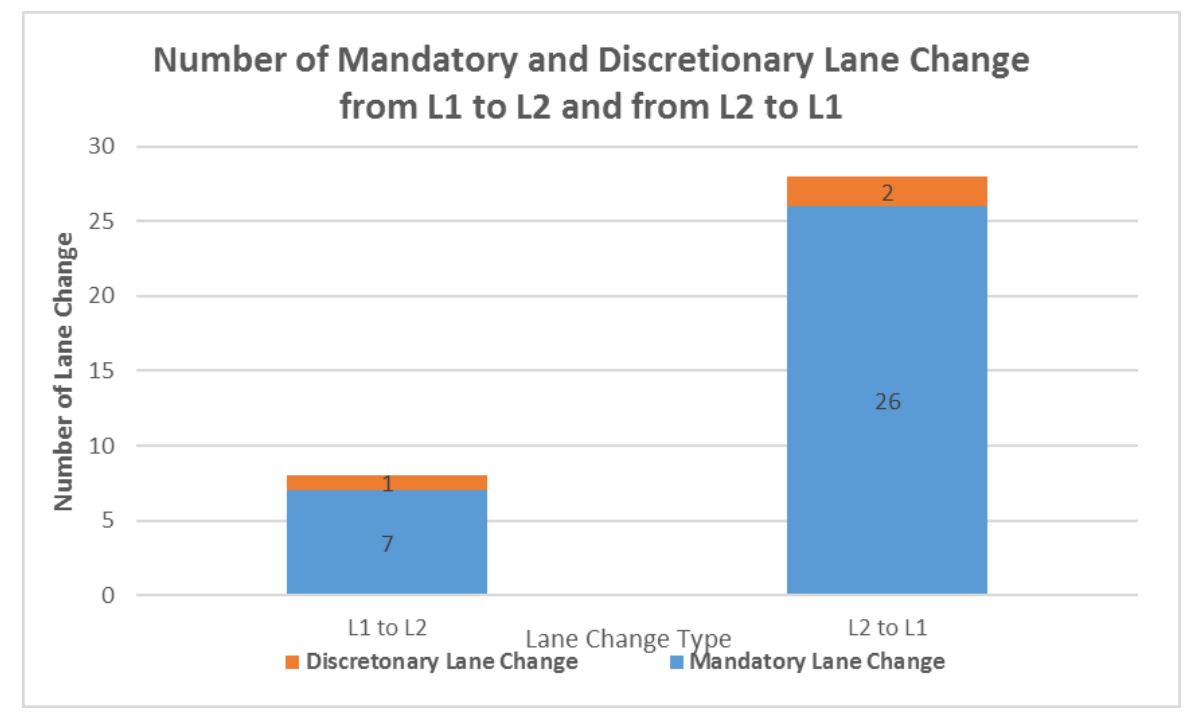

Fig. 8. Number of Mandatory and Discretionary Lane Change from L1 to L2 and from L2 to L1.

As illustrated in Error! Reference source not found. above, it can be observed that drivers are more likely to change lane to reach their destination (mandatory lane change) in urban condition. The main factor of this phenomenon is the high density of vehicles in Cell 7 resulting the space gap required for a subject vehicle to move to target lane is very small. In addition, the vehicles are tending to travel below free flow speed due to the high occupancy urban road. Therefore, there is low possibility that the drivers perform discretionary lane change in urban arterials to attain speed advantages. 


\section{Conclusion and Recommendations}

In this research, macroscopic traffic simulation based on Cell Transmission Model (CTM) is developed to model lane changing execution of two lane one-way urban arterials. A merging and diverging formula with pre-determined lane change rate were adopted to determine the number of vehicles executing lane change from origin lane to target lane and the number of vehicles flow through along origin lane.

A series of experiments are performed to evaluate the influences of lane change on delay and queue length. Three scenarios location specific lane change with various lane change rate were evaluated, comprising the driver changes lane as soon as possible at first cell, the driver desires to change lane increase linearly along the link and lastly the driver wishes to change lane at the last cell. Further analysis was done to study the relationship of lane density with mandatory and discretionary lane change occurrences.

The following conclusions are drawn throughout the research:

(1) Lane changing behavior approaching the exit of an intersection incurs highest delay and queue length.

(2) Number of lane change increases with the density in origin lane with a constant density in target lane.

The identified macroscopic lane change behavior that causes least delay in the traffic flows can be used for developing a microscopic lane change assistance system for human drivers or lane change control system of automated vehicles $[14,15]$. Further study is required to provide a better framework for modelling lane changing behavior. The recommendations for future work are listed as follows:

(1) The Influence of varying traffic demand on Delay with constant lane change rate.

(2) Variable Length and Shape of Cells. CTM simulates traffic condition by dividing the road network into equal length cell. Therefore, the length and shape of cells are sensitive parameters that influence the accuracy of CTM. A shorter cell length yields a more accurate result but requires much computational effort and vice versa. The cells might have variable cell shapes that are best aligned with the geometry. For instance, the shape of a cell that accumulate the queued left turns and right turns could be designed as a curve shape.

(3) Speed and Gap Acceptance Criteria. The speed of subject vehicles with preceding and following vehicles and gap acceptance criteria play a vital role in lane change decision. These two components should be further investigated to determine the minimum gap to accommodate the subject vehicles that wishes to change lane. Further analysis is required to examine the aggressiveness of driver lane changing behavior in oversaturated arterials.

This research has been made possible through financial contributions from the Ministry of Education Malaysia (MOHE) under Fundamental Research Grant Scheme (FRGS) [Project code FRGS/1/2015/TKO8/MUSM/03/1].

\section{References}


1. K.I. Ahmed, Modeling Drivers' Acceleration and Lane Changing Behavior, MIT, Dpt. C\&E Eng. (1999)

2. S. Ahn, M.J. Cassidy, 'Freeway traffic oscillations and vehicle lane-change maneuvers,' Transportation and Traffic Theory. Papers Selected for Presentation at ISTTT17 (2007)

3. R. Arnott, A bathtub model of downtown traffic congestion, J. Urban Econ., 76, pp. 11021 (2013)

4. J. Barceló, Models, traffic models, simulation, and traffic simulation, in Fundamentals of traffic simulation, Springer, pp. 1-62 (2010)

5. M. Carey, C. Balijepalli, D. Watling, 'Extending the Cell Transmission Model to Multiple Lanes and Lane-Changing', J. Infra. Modeling and Computation, 15, 3, pp. 50735 (2015)

6. M.J.Cassidy, J. Rudjanakanoknad, 'Increasing the capacity of an isolated merge by metering its on-ramp', Trans. Res. Pt. B, 39, 10, pp. 896-913 (2005)

7. R. Cheu, J. Martinez, C. Duran, 'A Cell Transmission Model with Lane Changing and Vehicle Tracking for Port of Entry Simulations', T.R.R.: J. TRB, 2124, pp. 241-8 (2009)

8. J.A. Cirillo, 'The relationship of accidents to length of speed-change lanes and weaving areas on Interstate highways', Highway Research Record, no. 312 (1970)

9. B. Coifman, R. Mishalani, C. Wang, S. Krishnamurthy, 'Impact of lane-change maneuvers on congested freeway segment delays: Pilot study', T.R.R.: J. TRB, no. 1965, pp. 152-159 (2006)

10. Gil Sander, F.B.M, C. Luis; R. Westra, Malaysia Economic Monitor: Transforming Urban Transport (2015)

11. L Immers, S. Logghe, 'Traffic flow theory', Faculty of Engineering, Department of Civil Engineering, Section Traffic and Infrastructure, Kasteelpark Arenberg, 40, p. 21 (2002)

12. R. Jiang, Q. Wu, Z. Zhu, 'A new dynamics model for traffic flow', Chinese Science Bulletin, 46, 4, pp. 345-8 (2001)

13. W.L Jin, 'Macroscopic Characteristics of Lane-Changing Traffic', Transportation Research Record: Journal of the Transportation Research Board, 2188, pp. 55-63 (2010)

14. M.A.S. Kamal, S. Taguchi, T. Yoshimura, 'Efficient driving on multilane roads under a connected vehicle environment', IEEE Transactions on Intelligent Transportation Systems, 17,9, pp. 2541-2551 (2016)

15. A. Kesting, M. Treiber, D. Helbing, General lane-changing model MOBIL for carfollowing models, T.R.R.: J. TRB, (1999), pp.86-94 (2007)

16. U. Khan, P. Basaras, L. Schmidt-Thieme, A. Nanopoulos, D. Katsaros, 'Analyzing cooperative lane change models for connected vehicles', in Connected Vehicles and Expo (ICCVE), 2014 International Conference on, pp. 565-70 (2014)

17. V. Knoop, S. Hoogendoorn, Y. Shiomi, C. Buisson,'Quantifying the number of lane changes in traffic: Empirical analysis', Transportation Research Record: Journal of the Transportation Research Board, no. 2278, pp. 31-41 (2012)

18. A. Kusuma, R. Liu, C. Choudhury, F. Montgomery, 'Lane-changing characteristics at weaving section', in Transportation Research Board 94th Annual Meeting (2015)

19. J.A. Laval, C.F. Daganzo, 'Lane-changing in traffic streams. (Report)', Transportation Research Part B: Methodological, 40, 3, p. 251 (2006)

20. M.J. Lighthill, G.B. Whitham, 'On Kinematic Waves. II. A Theory of Traffic Flow on Long Crowded Roads', Proceedings of the Royal Society of London. Series A, Mathematical and Physical Sciences (1934-1990), 229, no. 1178, pp. 317-45 (1955)

21. HCM, Highway capacity manual, Washington, DC (2010)

22. J. Milton, F. Mannering, 'The relationship among highway geometrics, traffic-related elements and motor-vehicle accident frequencies', Transportation, 25, 4, pp. 395-413 (1998) 
23. P. Wang, 'Conditional cell transmission model for two-way arterials in oversaturated conditions', The University of Alabama TUSCALOOSA (2010)

24. Q. Yang, H.N. Koutsopoulos, 'A microscopic traffic simulator for evaluation of dynamic traffic management systems', Transportation Research Part C: Emerging Technologies, 4,3, pp. 113-29 (1996)

25. F. Zhu, H.K. Lo, H.Z. Lin, Delay and emissions modelling for signalised intersections, Transportmetrica B: transport dynamics, 1,2, pp. 111-35 (2013) 